\title{
The effect of novel heterocyclic compounds
}

\section{on cryptococcal biofilm}

Maya Korem, Sarah Kagan and Itzhack Polacheck

Department of Clinical Microbiology and Infectious Diseases,

Hebrew University - Hadassah Medical Center, Jerusalem, Israel

\section{Background}

Biofilm formation by microorganisms depends on their communication by quorum sensing, which is mediated by small diffusible signaling molecules that accumulate in the extracellular environment. During human infection, the pathogenic yeast Cryptococcus neoformans can form biofilm on medical devices, which protects the organism and increases its resistance to antifungal agents. The aim of this study was to test two novel heterocyclic compounds, S8 (thiazolidinedione derivative, TZD) and NA8 (succinimide derivative, SI), for their anti-biofilm activity against strains of Cryptococcus neoformans and Cryptococcus gattii in a microtiter plate model

\section{Results}

\section{The effect on biofilm metabolic activity}

Treatment of $C$. neoformans $H-99$ and $C$. gattii $R-$ 272, with 58 or NA8 compounds during biofilm formation, reduces the biofilm metabolic activity (XTT)

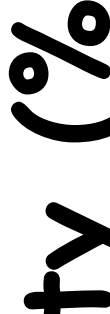

C. neoformans $H-99$

妾

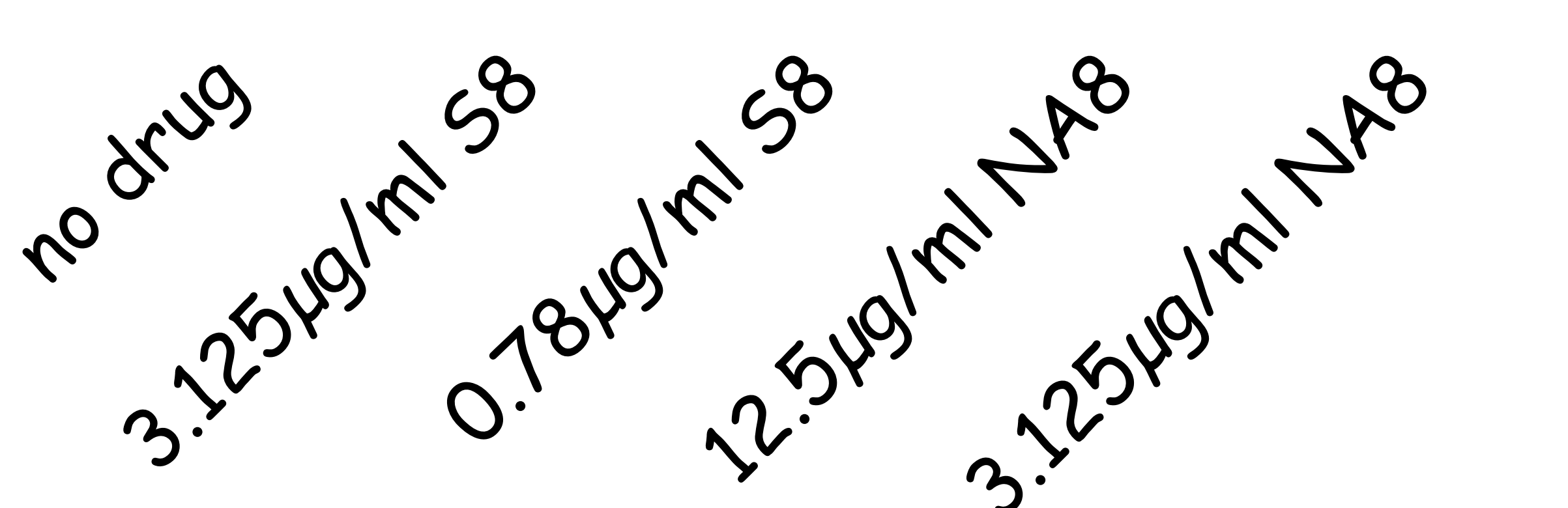

C. gattii $R-272$

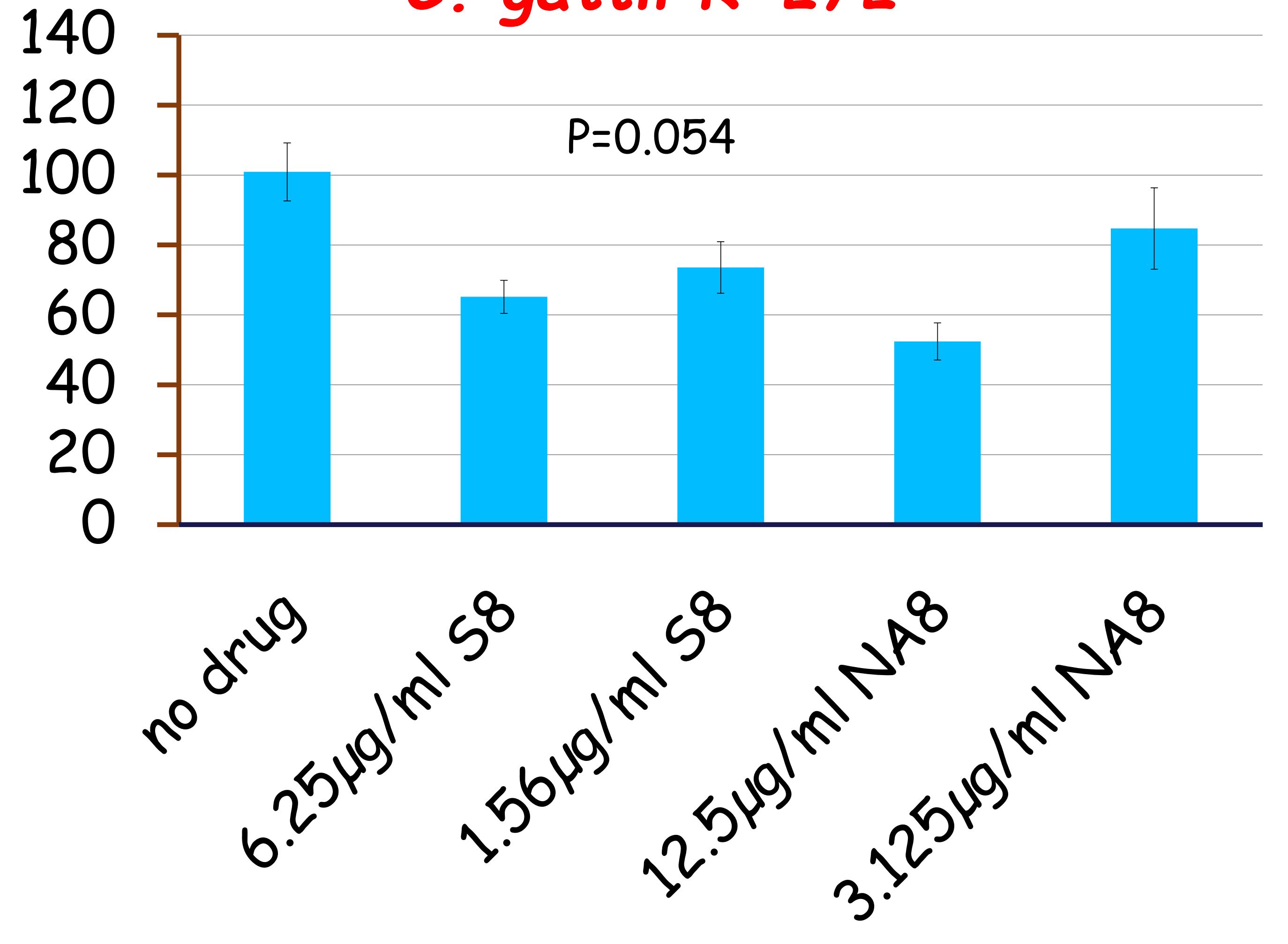

\section{Methods}

C. neoformans strain $\mathrm{H}-99$ (serotype A)

C. gattii strain R-272 (serotype B)

$30 C$ in a rotary shaker to early stationary phase

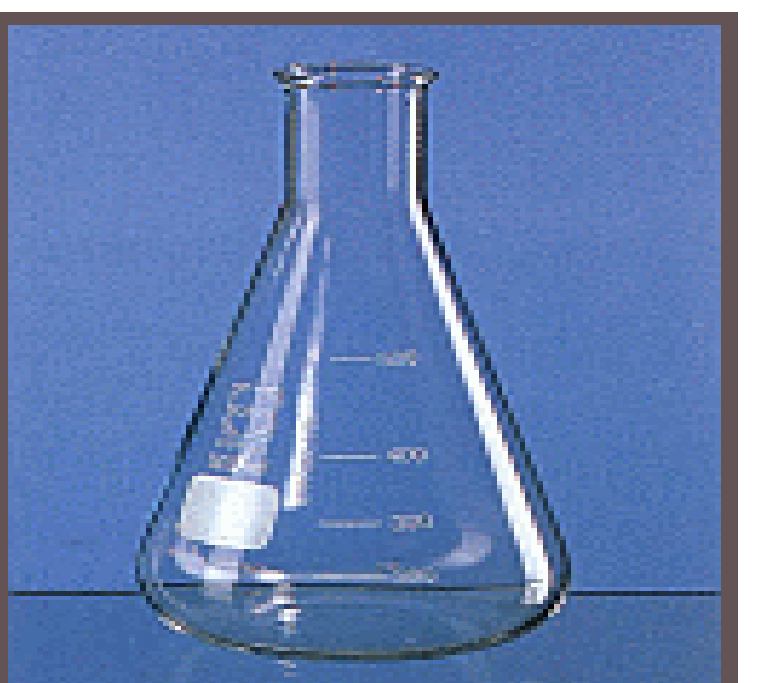

Biofilm formation medium

888888888

\begin{tabular}{|c|c|c|}
\hline \multirow{2}{*}{$\begin{array}{c}\text { Tested } \\
\text { compunds }\end{array}$} & \multicolumn{2}{|c|}{ MIC $(\mu \mathrm{g} / \mathrm{ml})$} \\
\hline & C. neoformans & C. gattii \\
\hline 58 & 12.5 & 25 \\
\hline NA8 & 50 & 50 \\
\hline
\end{tabular}

XTT metabolic reduction assay method measuring method measuring activity of cells)

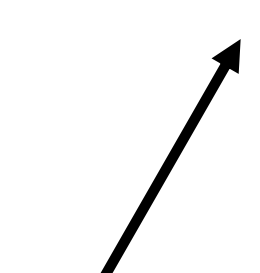

$48 \mathrm{~h}$ incubation $35^{\circ} \mathrm{C}$ ambient $\mathrm{CO} 2$
determined MIC
than washed $\begin{array}{cc}\mathrm{CO} 2 \\ \text { determined MIC } & \text { than washed }\end{array}$
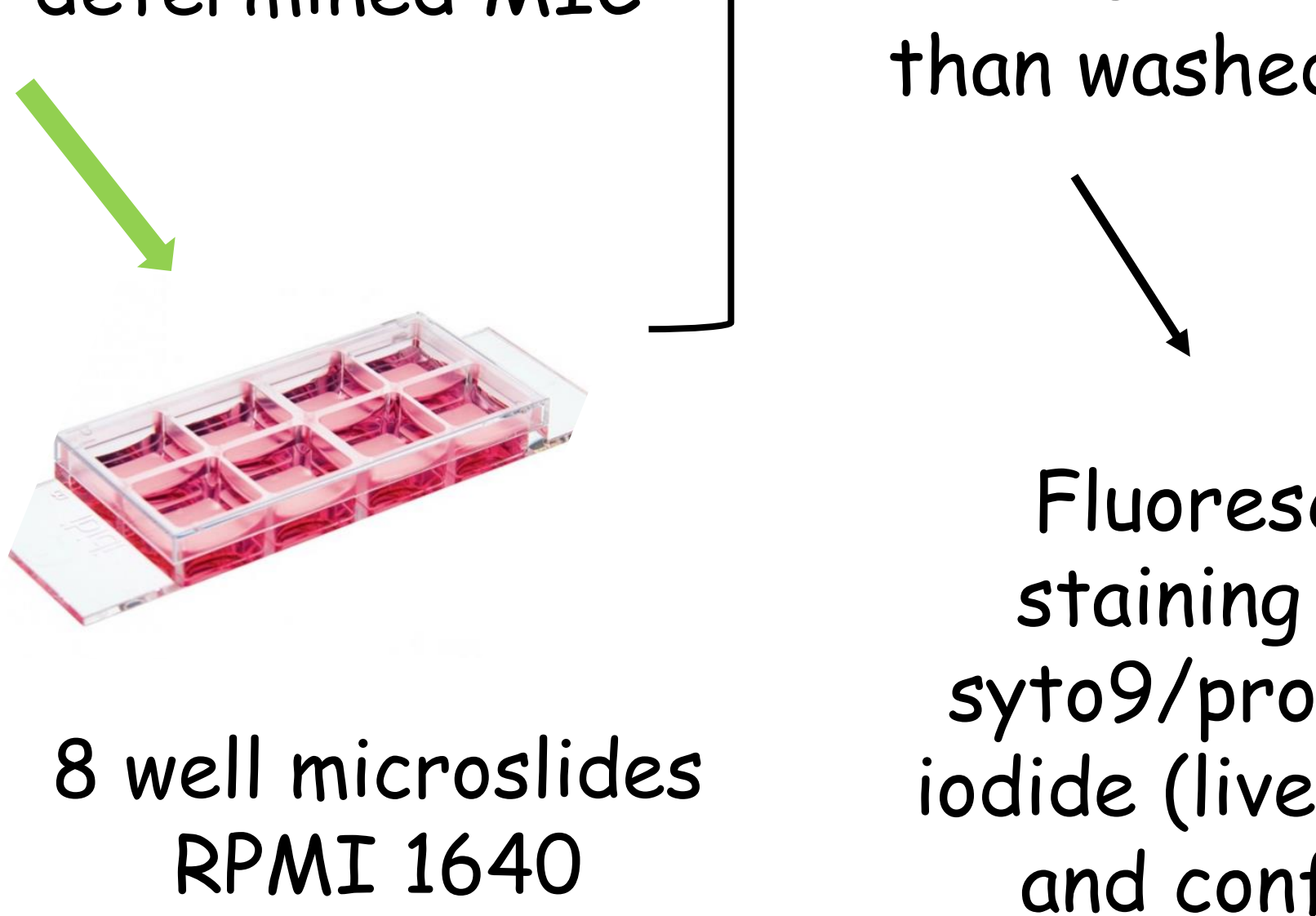

Fluorescent staining with syto9/propidium iodide (live/ dead) and confocal analysis

\section{Confocal microscopy}

Staining with live/dead dye (Syto9/propidium iodide), and analysis of biofilm thickness by confocal microscopy. Green and Yellow/Red represent live and dead yeast, respectively.

C. neoformans H-99

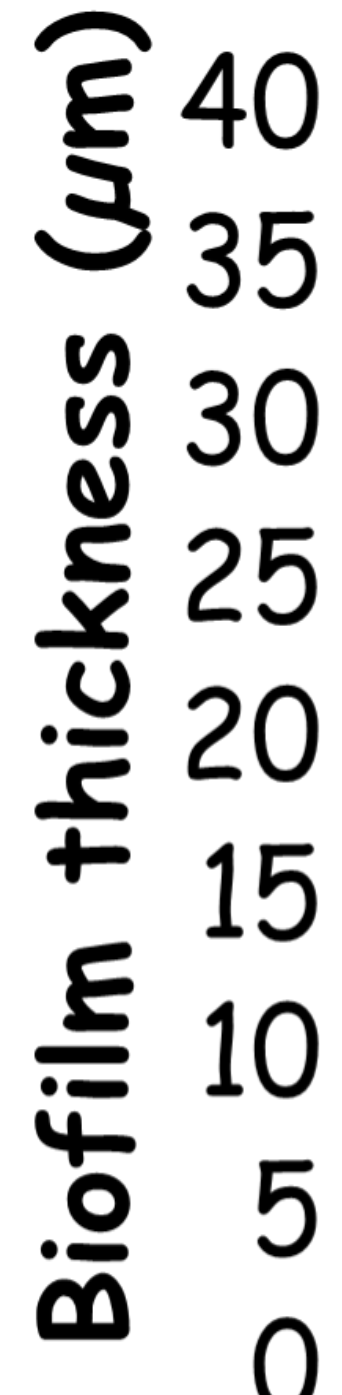

NA8 and 58 reduce nucleic acid staining by Syt09. 58 also causes yeast death in the biofilm
C. gattii $R-272$

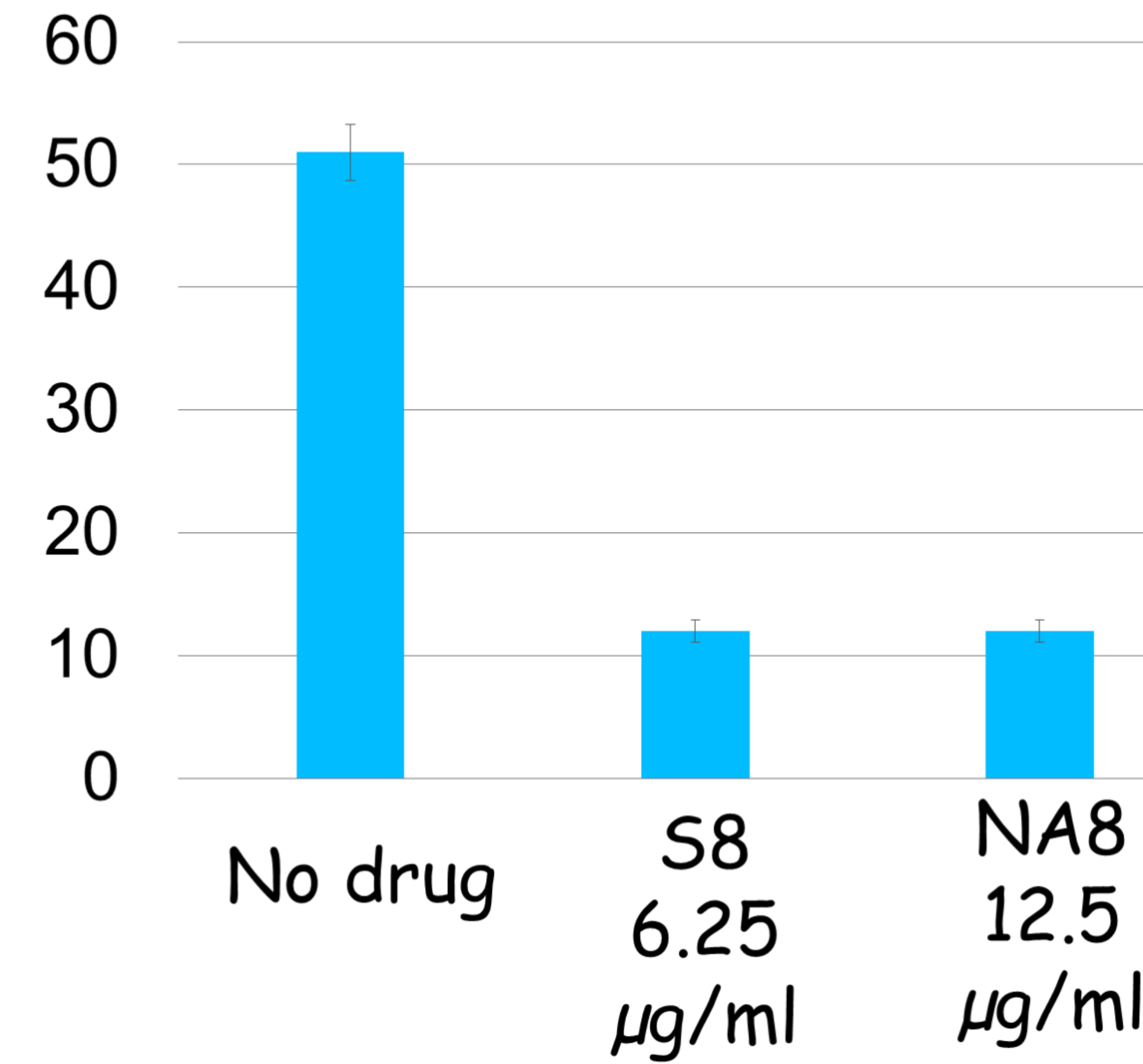

\section{Summary and conclusions}

58 and NA8 reduce nucleic acid staining by Syto 9

$\mathrm{NA} 8-12.5 \mu \mathrm{g} / \mathrm{ml} \quad \mathrm{S} 8-3.125 \mu \mathrm{g} / \mathrm{ml}$

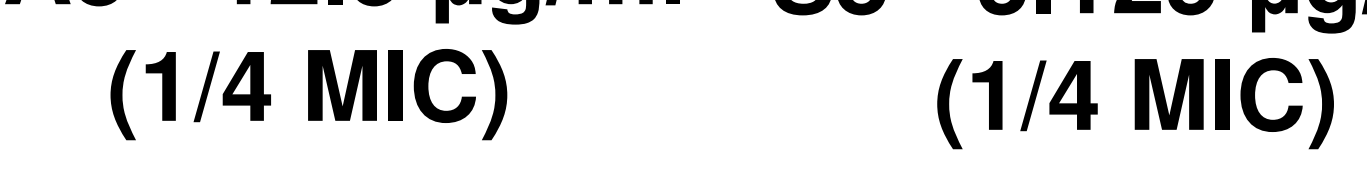

No drug NA8 $-12.5 \mu \mathrm{g} / \mathrm{ml} \quad \mathrm{S} 8-6.25 \mu \mathrm{g} / \mathrm{ml}$ No drug
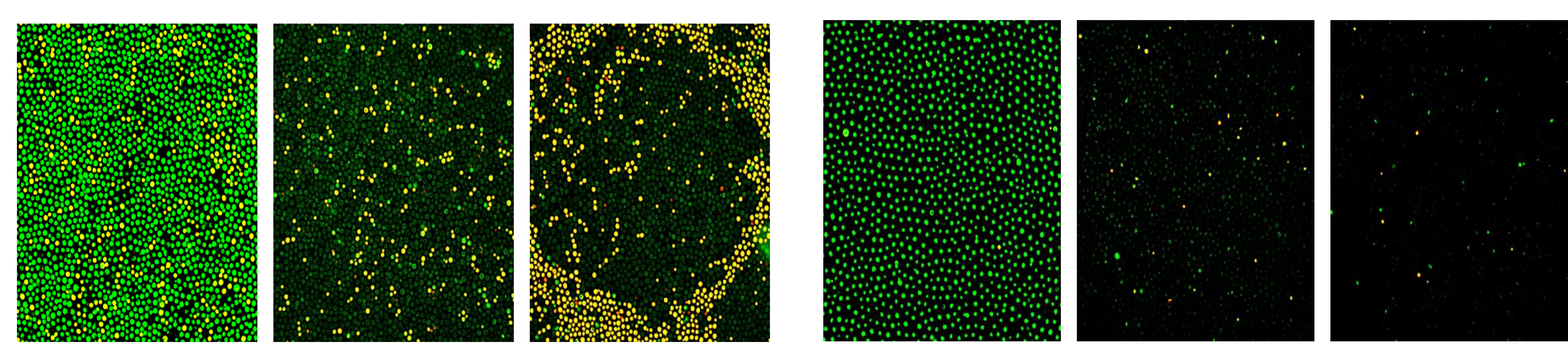

Cryptococcus neoformans/gattii Planktonic cells

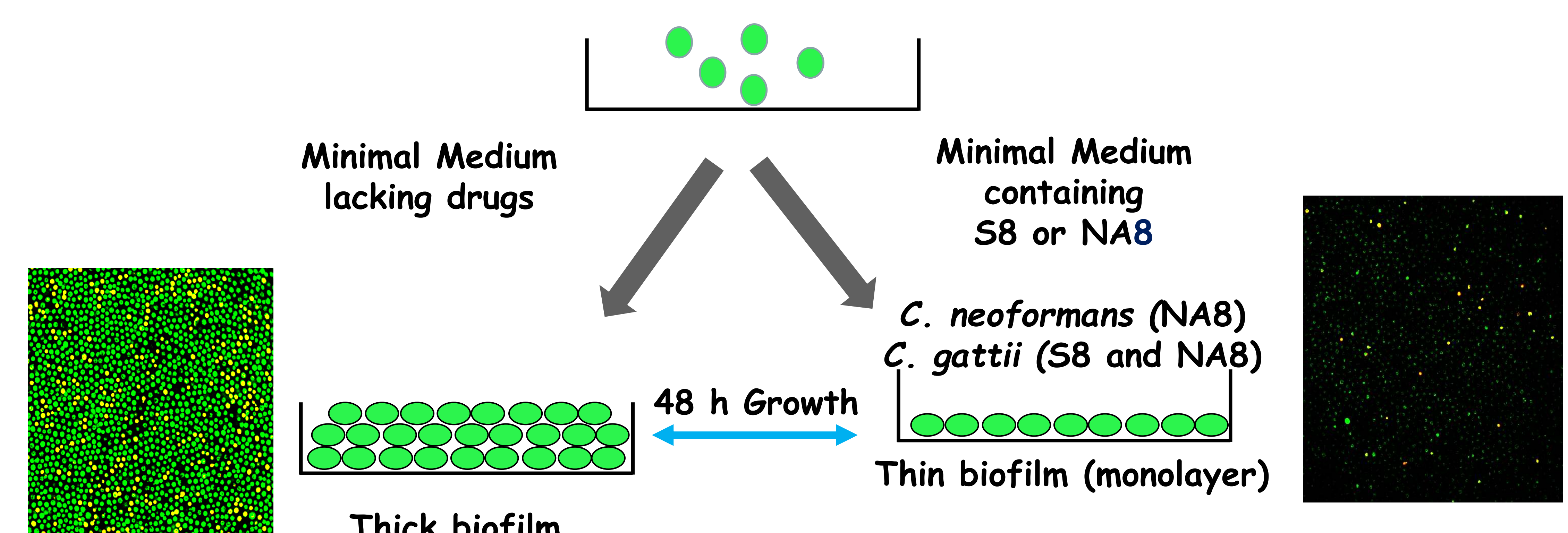

The formation of biofilm by $C$. neoformans and $C$. gattii on the polystyrene plates was inhibited significantly by two novel heterocyclic compounds in concentrations below the MIC value.

Our results indicate that the development of cryptococcal biofilms may be prevented by 58 and NA8 heterocyclic compounds, suggesting the possibility of preventive or even therapeutic measures against fungal biofilm development on indwelling medical devices. 